\title{
EZH2 is overexpressed in laryngeal squamous cell carcinoma and enhances the stem-like properties of AMC-HN-8 cells
}

\author{
JIAMENG HUANG ${ }^{1}$, LIANG ZHOU ${ }^{1}$, HUI CHEN ${ }^{1}$, CHUNPING WU $^{1}$, ZHANG DUO $^{1}$ and YANPING ZHANG ${ }^{2}$ \\ ${ }^{1}$ Department of Otolaryngology-Head and Neck Surgery; ${ }^{2}$ Research Center, \\ Affiliated Eye, Ear, Nose and Throat Hospital of Fudan University, Shanghai 200031, P.R. China
}

Received March 19, 2015; Accepted May 3, 2016

DOI: $10.3892 / \mathrm{ol} .2016 .4704$

\begin{abstract}
The enhancer of zeste 2 polycomb repressive complex 2 subunit (EZH2) histone methyltransferase is the catalytic subunit of polycomb repressive complex 2 (PRC2), which is important for epigenetic regulation. EZH2 is highly expressed in various types of tumors, and its high-level expression promotes the progression and invasion of certain tumors. However, the expression level of EZH2 and its functions in laryngeal squamous cell carcinomas are unknown. In the present study, the level of EZH2 expression in laryngeal squamous cell carcinomas was evaluated using immunochemical staining and reverse transcription-quantitative polymerase chain reaction. EZH2 was overexpressed in AMC-HN-8 cells with lentiviral transfection. Cell proliferation, apoptosis, cell-cycle, chemotherapy-sensitivity and in vivo tumorigenic assays were performed. The results indicated that EZH2 was highly expressed in laryngeal squamous cell carcinomas. Additionally, EZH2 overexpression promoted proliferation, accelerated cell-cycle progression and enhanced the tumorigenicity in laryngeal squamous cancer cells. More importantly, EZH2 enhanced the chemotherapy resistance of these cells. Overall, the results indicated that EZH2 promotes the progression of laryngeal squamous cell cancer and could be a potential chemotherapeutic target for the treatment of such cancer.
\end{abstract}

\section{Introduction}

Laryngeal squamous cell carcinoma is the second most common malignant squamous cell carcinoma of the head and neck (1). Although the 5-year survival rate has been

Correspondence to: Professor Liang Zhou, Department of Otolaryngology-Head and Neck Surgery, Affiliated Eye, Ear, Nose and Throat Hospital of Fudan University, 83 Fenyang Road, Shanghai 200031, P.R. China

E-mail: zhoulent@126.com

Key words: enhancer of zeste 2 polycomb repressive complex 2 subunit, laryngeal squamous cell carcinoma, expression, stem-like properties, AMC-HN-8 cell line increased to a high level, numerous patients succumb to local recurrence, regional recurrence or distant metastasis following surgery (1). Therefore, it is important to discover the molecular mechanisms underlying the development of laryngeal squamous cell carcinomas. Enhancer of zeste 2 polycomb repressive complex 2 subunit (EZH2) is the catalytic subunit of polycomb repressive complex 2 (PRC2), which is a highly-conserved histone methyltransferase that targets lysine-27 of histone H3 (2). EZH2 contains a signature SET domain, which provides the methyltransferase active site. The interaction of EZH2 with DNA methyltransferases (DNMTs) results in the transcriptional repression of target genes (3). EZH2 has been demonstrated importance in the development of human embryonic stem cells (4). Ezhkova et al (5) reported that EZH2 regulates the proliferative capacity of epidermal progenitor cells by suppressing the Ink4A-Ink4B locus, and moderates their differentiation by preventing the early recruitment of the jun proto-oncogene transcriptional activator to the structural genes required for epidermal differentiation. In addition, EZH2 has been shown to be highly expressed in cancer cells, particularly in stem cell-like cancer cell lines, in numerous cancer models $(6,7)$. However, the expression and function of EZH2 have been seldom investigated in laryngeal squamous cell carcinomas, and the role of EZH2 in laryngeal carcinoma is currently unknown. The present study systematically evaluated the expression of EZH2 in laryngeal carcinomas and investigated the functions of EZH2 in laryngeal cancer cells in vitro and in vivo.

\section{Materials and methods}

Ethics statement. The tumor specimens used in the present study were obtained with the approval of the ethics committee of the Eye, Ear, Nose and Throat Hospital of Fudan University, Shanghai, China. Signed informed consent was obtained from each patient. The animal care and experimental protocols were approved by the Shanghai Medical Experimental Animal Care Committee, Shanghai, China. Animals were fed in laminar flow cabinets at the Department of Laboratory Animal Science of Fudan University under specific pathogen-free conditions. All of the surgeries were performed under ketamine/xylazineinduced anesthesia, and all efforts were made to minimize suffering. 
Patients and tissue specimens. All the surgical tissue specimens were obtained from patients with laryngeal squamous cell carcinomas, who had previously received surgical treatment at the Eye, Ear, Nose and Throat Hospital of Fudan University, between March 2012 and December 2013. No patient had received chemotherapy or radiotherapy. A total of 80 primary tumor tissues and the corresponding paracancerous epithelial tissues were stored in paraformaldehyde at room temperature. An additional 25 primary tumor tissues and the corresponding paracancerous epithelial tissues were stored at $-80^{\circ} \mathrm{C}$. Tissues were categorised using the most recent ( 7 th) edition of the tumor, node, metastasis (TNM) system of classification defined by the International Union Against Cancer (8)

Tissue microarray construction. Samples of 80 tumor tissues and 80 paracancerous epithelial tissues were selected for the construction of the tissue microarray. All samples were fixed with 4\% paraformaldehyde (Wuhan Boster Biological Technology, Ltd., Wuhan, China), embedded in paraffin (Wuhan Boster Biological Technology, Ltd.), cut to the desired thickness of $3 \mu \mathrm{m}$, and affixed to slides. The slides were stained with hematoxylin and eosin (Wuhan Boster Biological Technology, Ltd.) and assessed by two histopathologists. Two representative tissue cores of each tissue block were selected for transfer to a master block using a manual tissue microarray instrument (ATA-27; Beecher Instruments, Inc., Sun Prarie, WI, USA). The master block was cut to the desired thickness of $4 \mu \mathrm{m}$, and sections were placed on 3-aminopropyltriethoxysilane-coated slides (Wuhan Boster Biological Technology, Ltd.).

Immunohistochemistry. The tissue microarray section was deparaffinized with xylene, rehydrated with a graded series of ethanol solutions, rinsed with phosphate-buffered saline (PBS), and treated with $3 \%$ hydrogen peroxide to inactivate the endogenous peroxidases. The section was then treated with boiling water containing ethylenediaminetetraacetic acid (EDTA; Invitrogen; Thermo Fisher Scientific, Inc., Waltham, MA, USA) for $5 \mathrm{~min}$ to retrieve the epitopes. Subsequent to washing the section 3 times with PBS and incubating it with the primary antibody (anti-KMT6/EZH2 antibody-ChIP grade; rabbit polyclonal; dilution, 1:100; catalog no., ab3748; Abcam, Cambridge, MA, USA) for $12 \mathrm{~h}$, the section was washed 3 times with PBS and incubated with a goat antirabbit secondary antibody (dilution, 1:250; cat no., BA1003; Wuhan Boster Biological Technology, Ltd, Shanghai, China) for 1 h. 3,3'-diaminobenzidine (Wuhan Boster Biological Technology, Ltd.) acted as the chromogen. Images were captured with a fluorescence microscope (DMI4000b; Leica, Wetzlar, Germany) and were assessed by two pathologists. The standard for evaluation was as follows: Percentage of positive cells was scored $0,0 \%$ positive cells; $1,1-10 \%$ positive cells; 2 , $11-50 \%$ positive cells; and $3,>50 \%$ positive cells; the staining intensity was scored 0 , negative; 1 , weak; 2 , moderate; and 3, high. The final score was the sum of the two scores. A score of 0-3 was considered negative, and a score of 4-9 was considered positive (9).

Reverse transcription (RT)-quantitative polymerase chain reaction $(q P C R)$. Total RNA was extracted from the following samples using TRIzol (Invitrogen; Thermo Fisher Scientific,
Inc.): Freshly frozen tissue samples, including 25 tumor tissue and 25 corresponding paracancerous epithelial tissue samples; AMC-HN-8 cells; and EZH2-overexpressing AMC-HN-8 cells. RNA $(1 \mu \mathrm{g})$ was reverse-transcribed into complementary DNA (cDNA) using the PrimeScript RT Master Mix, which included PrimeScript RTase, RNase inhibitor, Random 6 mers, Oligo dT Primer, dNTP Mixture and buffer (catalog no., RR036Q; Takara Biotechnology Co., Ltd., Dalian, China). The protocol included $37^{\circ} \mathrm{C}$ for $15 \mathrm{~min}$, followed by $85^{\circ} \mathrm{C}$ for $5 \mathrm{sec}$ and $4^{\circ} \mathrm{C}$ for a sustained period of time. RT-qPCR was performed with SYBR Premix Ex Taq ${ }^{\mathrm{TM}}$ (Takara Biotechnology Co., Ltd.) and an ABI7500 instrument (Applied Biosystems; Thermo Fisher Scientific, Inc.), according to the manufacturer's instructions (initial denaturation for $30 \mathrm{sec}$ at $95^{\circ} \mathrm{C}$, then 40 cycles of $95^{\circ} \mathrm{C}$ for $5 \mathrm{sec}$ and $60^{\circ} \mathrm{C}$ for $34 \mathrm{sec}$ ). Glyceraldehyde 3-phosphate dehydrogenase (GAPDH) was selected as the control. The following primers were used for PCR: EZH2 forward primer (5'-3'), GCCAGACTGGGAAGAAATCTG and EZH2 reverse primer (5'-3'), TGTGCTGGAAAATCCAAGTCA; GAPDH forward primer, (5'-3') CGGAGTCAACGGATTTGGTCG TAT and GAPDH reverse primer, (5'-3') AGCCTTCTCCAT GGTGGTGAAGAC (Sangon Biotech Co., Ltd., Shanghai, China). The level of EZH2 messenger RNA (mRNA) expression was calculated using the $2^{-\Delta \Delta \mathrm{Cq}}$ method.

Cell culture. The laryngeal squamous cell cancer AMC-HN-8 cell line was obtained from the Central Laboratory of the Eye, Ear, Nose and Throat Hospital of Fudan University. The AMC-HN-8 cell line was cultured in RPMI-1640 medium (Gibco; Thermo Fisher Scientific, Inc.) containing 10\% fetal bovine serum (FBS; Invitrogen; Thermo Fisher Scientific, Inc.) and $1 \%$ penicillin/streptomycin (Invitrogen; Thermo Fisher Scientific, Inc.) in a humidified incubator at $5 \% \mathrm{CO}_{2}$ and $37^{\circ} \mathrm{C}$. The medium was replaced every 2 days.

Construction of the EZH2 overexpression lentiviral vector and transfection. Information regarding the lentiviral EZH2 overexpression vector system (Fig. 1) is described below. The primer sequences were as follows: Human EZH2-F (Xhol+Flag), CCGCTCGAGGCCACCATGGGCCAGACT GGGAAGAA; and human EZH2-R (BamHI), CCGGGA TCCTCAAGGGATTTCCATTTCTCTT. The primers were synthesized at Sangon Biotech Co., Ltd.. After the DNA was linked with the vector using DNA ligase subsequent to double enzyme digestion (Fig. 1A), the plasmid was transfected into 293T competent cells. The supernatant was collected following ultracentrifugation at 4,500 $\mathrm{x}$ g. The virus titer was determined using flow cytometry (BD FACSCalibur; BD Biosciences, Franklin Lakes, NJ, USA). Finally, the EZH2-overexpressing lentiviruses were transfected into AMC-HN-8 cells in the aforementioned conditions of cell culture for 1 day. A stably transfected cell line overexpressing EZH2 was established after selection using puromycin. RT-qPCR (using the same protocol as above) and western blotting were performed to determine the degree of overexpression.

Western blotting. Total protein was extracted from AMC-HN-8 cells and EZH2-overexpressing cells with radioimmunoprecipitation assay lysis and extraction buffer (Beyotime Institute of Biotechnology, Haimen, China). Western 
blotting was performed as previously described (10) using an anti-KMT6/EZH2 antibody (rabbit polyclonal; dilution, 1:1,000; catalog no., ab3748; Abcam) and a mouse anti-human $\beta$-actin antibody (dilution, 1:1,000; catalog no., A5441; Sigma-Aldrich, St. Louis, MO, USA). The secondary antibody was a goat anti-rabbit $\mathrm{IgG}$ horseradish peroxidase-conjugated antibody (dilution, 1:2,000; cat no. sc-2030; Santa Cruz Biotechnology, Inc., Dallas, TX, USA). The membrane was developed using an enhanced chemiluminescent substrate (Thermo Fisher Scientific, Inc.).

Cell proliferation assay. AMC-HN-8 cells and the EZH2-overexpressing AMC-HN-8 cells were cultured in a 96-well plate (Corning Incorporated, Corning, NY, USA) at a density of $1 \times 10^{3}$ cells/well in $0.1 \mathrm{ml}$ RPMI-1640 medium, containing $10 \% \mathrm{FBS}$ in a humidified incubator at $5 \%$ $\mathrm{CO}_{2}$ and $37^{\circ} \mathrm{C}$. The medium was changed every 2 days. The wells containing each cell line were divided into 4 groups, according to the day on which the cell proliferation assay was performed as follows: Day 1, 3, 5 and 7. Each group contained 6 replicate wells. cell counting kit-8 reagent $(\mathrm{CCK}-8 ; 10 \mu \mathrm{l}$; Dojindo Molecular Technologies, Inc., Kumamoto, Japan) was added to the wells, and the plate was incubated for $2.5 \mathrm{~h}$. The absorbance of each sample at $450 \mathrm{~nm}$ was determined using an ELISA microplate reader (Bio-Rad 680; Bio-Rad Laboratories, Inc., Hercules, CA, USA).

Apoptosis assay. AMC-HN-8 cells and EZH2-overexpressing cells were harvested by adding a trypsin solution without EDTA (Gibco; Thermo Fisher Scientific, Inc.) followed by the addition of medium containing FBS to terminate the trypsin activity. The cells were centrifuged at $800 \mathrm{x} g$ and washed twice with PBS. The cells were incubated with fluorescein isothiocyanate-conjugated anti-annexin V (Invitrogen; Thermo Fisher Scientific, Inc.) and propidium iodide (PI; Invitrogen; Thermo Fisher Scientific, Inc.), and the rates of apoptosis were determined using flow cytometry (BD FACSCalibur: BD Biosciences, Inc, Franklin Lakes, NJ, USA). The results were analyzed using FlowJo 7.6 (FlowJo, LLC, Ashland, OR, USA).

Cell cycle assay. AMC-HN-8 cells and EZH2-overexpressing cells $\left(1 \times 10^{6}\right)$ were washed twice with PBS and were centrifuged at $800 \mathrm{x} \mathrm{g}$. The cells were then suspended in $1 \mathrm{ml}$ of PBS, to which $9 \mathrm{ml}$ of $70 \%$ ethanol was slowly added while vortexing. The cells were maintained at $4^{\circ} \mathrm{C}$ overnight. Prior to flow cytometric analysis, the cells were washed twice with PBS, treated with $10 \mathrm{mg} / \mathrm{ml}$ RNase A (Sigma-Aldrich) for $30 \mathrm{~min}$, and stained with $50 \mu \mathrm{g} / \mathrm{ml} \mathrm{PI}$ in the dark for $1 \mathrm{~h}$. Each cell type was analyzed in triplicate.

Chemotherapy sensitivity assay. AMC-HN-8 cells and EZH2-overexpressing cells were cultured in a 96-well plate (Corning Incorporated) at a density of $1 \times 10^{4} / \mathrm{ml}$. The two types of cells were divided into 4 groups, according to the concentration of cisplatinum applied. Cisplatinum was applied to the groups at concentrations of 0, 3, 6 and $12 \mu \mathrm{g} / \mathrm{ml}$. Each group contained 6 replicate wells. Subsequent to incubation with cisplatinum for $24 \mathrm{~h}$, the number of cells in each well was determined using a CCK- 8 assay, following the aforementioned method. The growth inhibition rate was calculated using the following formula: Inhibition rate $=1-$ (mean absorbance of the test well) / (mean absorbance of the control well) x $100 \%$.

In vivo tumorigenicity test. Six non-obese diabetic (NOD) mice (4-weeks old, male) were purchased from Shanghai Super-B\&K Laboratory Animal Corp., Ltd. (Shanghai, China). AMC-HN-8 cells and EZH2-overexpressing cells were trypsinized, resuspended in RPMI-1640 medium at a density of $1 \times 10^{7} / \mathrm{ml}$, and then injected into the subcutaneous space of the axillary fossa of mice under ketamine/xylazine-induced anesthesia $\left(2 \times 10^{6}\right.$ cells/mouse). After 20 days, the NOD mice were sacrificed by cervical dislocation, and the tumors were removed surgically. The tumors were photographed, weighed, fixed with paraformaldehyde, stained with hematoxylin-eosin and examined.

Statistical analysis. All experiments were performed independently 3 times. All results, with the exception of the immunohistochemical results, were expressed as the mean values \pm standard deviation and were analyzed with the independent sample Student's $t$-test. The immunohistochemical results were analyzed using the $\chi^{2}$ test. IBM SPSS statistics version 20 (IBM SPSS, Armonk, NY, USA) was used to compare the statistical difference between EZH2 overexpression cells and AMC-HN-8 cells. A P-value of $<0.05$ was considered to indicate a statistically significant difference.

\section{Results}

EZH2 is expressed more highly in laryngeal squamous cell cancer tissues compared with paracancerous epithelial tissues. In the present study, RT-qPCR and immunohistochemical assays of primary laryngeal squamous cell cancer tissues and paracancerous epithelial tissues were performed to detect the expression of EZH2 at the transcriptional level and protein expression level, respectively. The total mRNA of 25 tumor tissues and 25 corresponding paracancerous epithelial tissues was extracted and then reverse transcribed into cDNA, which was utilized for qPCR. This quantitative analysis showed that the EZH2 mRNA expression level in the tumor tissues was significantly increased compared with paracancerous epithelial tissues ( $\mathrm{P}=0.0003$; Fig. 2). To determine whether EZH2 was highly expressed at the protein level in tumor tissues, 80 tumor tissue samples and 80 corresponding paracancerous epithelial tissue samples from 80 patients were used to prepare a tissue microarray for the immunohistochemical detection of EZH2 protein. Representative images are shown in Fig. 3. The tumor and paracancerous tissues expressed EZH2; however, the expression levels were significantly different between the two groups $(\mathrm{P}=0.0004)$. EZH2 was diffusely distributed throughout the primary tumor tissues, but was mainly expressed in the basal layer in the paracancerous tissue samples, which indicated that EZH2 may be involved in cell proliferation. By comparing the immunoreactive scores for the two types of tissues, EZH2 was found to be highly expressed in the tumor tissues, which supported the RT-qPCR results. To investigate the association between the level of EZH2 expression and the clinical characteristics of the laryngeal squamous cells cancers, the level of EZH2 expression in tumors of various clinical stages 

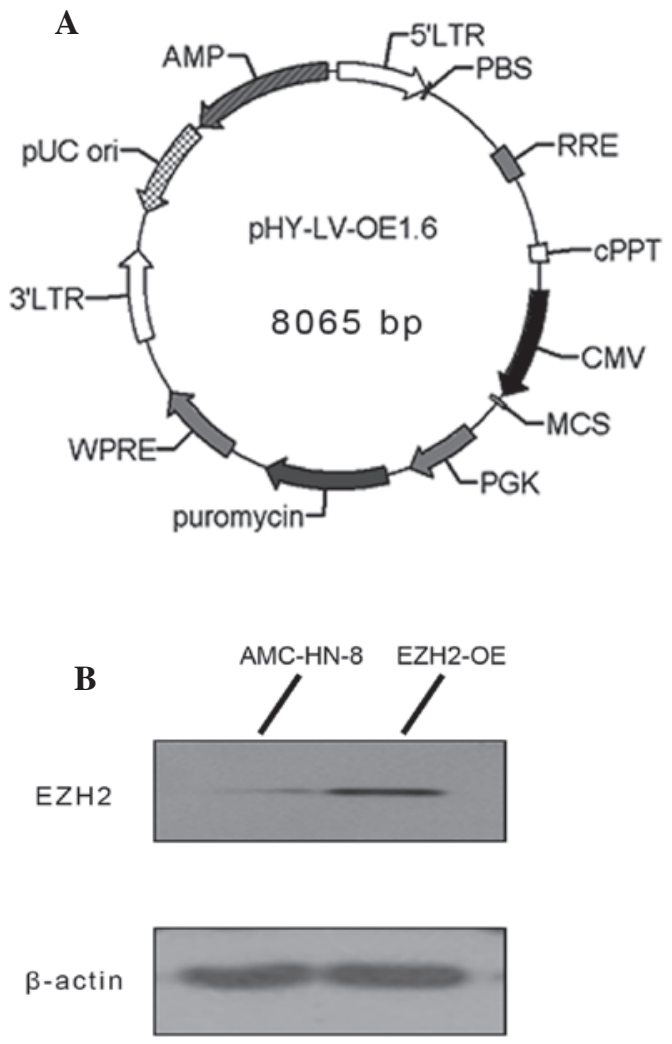

C

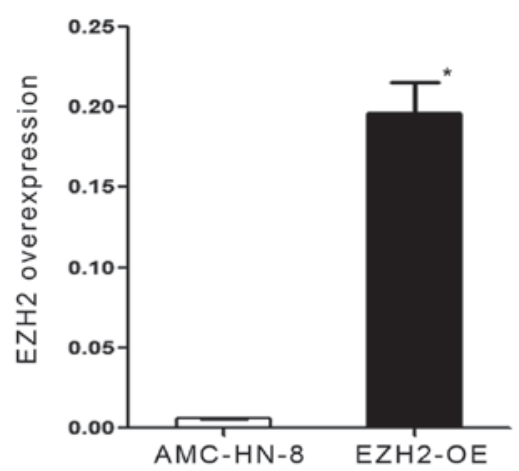

D

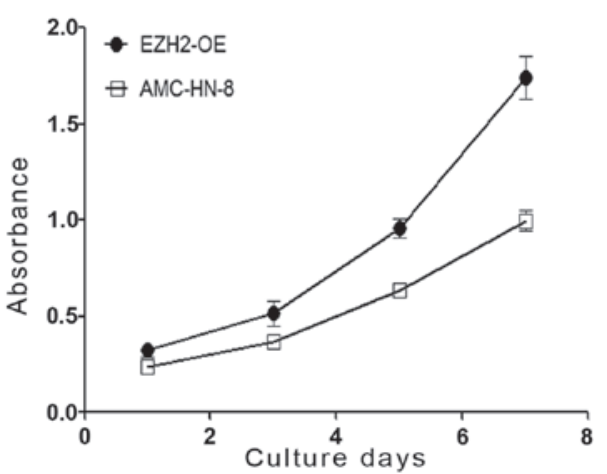

Figure 1.Lentiviral transfection and analysis of cell proliferation. (A) Diagram of the EZH2 overexpression lentiviral vector system. (B) Western blotting was used to determine the degree of EZH2 overexpression in the AMC-HN-8 cell lines. (C) Reverse transcription-quantitative polymerase chain reaction was used to determine the degree of EZH2 overexpression at the messenger RNA level in the AMC-HN-8 cell lines. (D) The proliferation assay was performed on the AMC-HN-8 cells and EZH2-OE cells on days 1,3,5 and 7 of culture using the cell counting kit- 8 method. The results were expressed as the mean values \pm standard deviation. ${ }^{*} \mathrm{P}<0.05$. EZH 2 , enhancer of zeste 2 polycomb repressive complex 2 subunit; EZH2-OE, EZH2-overexpressing.
Table I. Clinical indices and EZH2 expression.

\begin{tabular}{lccc}
\hline & \multicolumn{3}{c}{ EZH2 expression } \\
\cline { 2 - 4 } Clinical index & Negative & Positive & P-value \\
\hline TNM stage & 38 & 21 & 0.982 \\
I-II & 11 & 6 & \\
III-IV & 37 & 13 & 0.007 \\
Tumor location & 11 & 15 & \\
Glottic & & & \\
Nonglottic & 48 & 28 & $<0.001$ \\
Tissue type & 63 & 8 & \\
Tumor tissue & & & \\
Normal tissue & 63 &
\end{tabular}

$\chi^{2}$ test was used to analyze all of the data. EZH2, enhancer of zeste 2 polycomb repressive complex 2 subunit; TNM, tumor-node-metastasis.

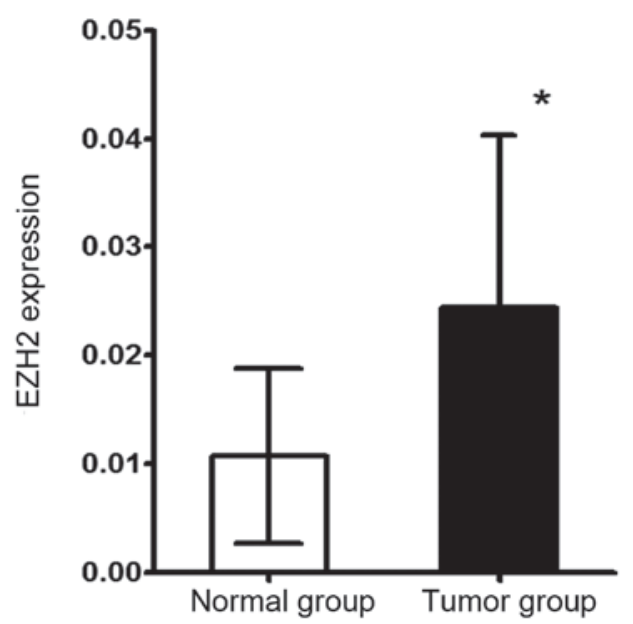

Figure 2. Reverse transcription-polymerase chain reaction was used to determine the level of EZH2 messenger RNA expression in 25 tumor tissues and the 25 corresponding paracancerous tissues. The results were expressed as the mean values \pm standard deviation. ${ }^{*} \mathrm{P}<0.05$. EZH2, enhancer of zeste 2 polycomb repressive complex 2 subunit.

and locations was compared. EZH2 was expressed more highly in glottic cancers compared with nonglottic cancers $(\mathrm{P}=0.007)$, but there was no significant difference in the levels of EZH2 expression between T1-T2 stage and T3-T4 stage tumors $(\mathrm{P}=0.982$; Table $\mathrm{I})$.

EZH2 overexpression stimulates the proliferation of $A M C-H N-8$ cells through promoting entry into the synthesis phase of the cell cycle. Through transfection of an EZH2 overexpression lentiviral vector, a cell line that stably overexpressed EZH2 was established. In this cell line, the transfection effects were confirmed by western blot analysis at protein level (Fig. 1B), the level of EZH2 expression was 30-fold greater compared with the control group ( $\mathrm{P}=0.011$; Fig. $1 \mathrm{C})$ and the efficacy of this expression was not reduced by passaging the cells. To investigate the role of EZH2 in the proliferation of 
Table II. Absorbance of the AMC-HN-8 cells and the EZH2-OE cells.

\begin{tabular}{lcccc}
\hline & \multicolumn{4}{c}{ Day } \\
\cline { 2 - 5 } Type & $1^{\mathrm{a}}$ & $3^{\mathrm{b}}$ & $5^{\mathrm{c}}$ & $7^{\mathrm{d}}$ \\
\hline EZH2-OE & $0.3242 \pm 0.0636$ & $0.5148 \pm 0.1100$ & $0.9573 \pm 0.0870$ & $1.7426 \pm 0.1932$ \\
AMC-HN-8 & $0.2365 \pm 0.0451$ & $0.3711 \pm 0.0235$ & $0.6345 \pm 0.0396$ & $0.9988 \pm 0.0925$ \\
\hline
\end{tabular}

${ }^{\mathrm{a}} \mathrm{P}=0.124 ;{ }^{\mathrm{b}} \mathrm{P}=0.147 ;{ }^{\mathrm{c}} \mathrm{P}=0.012 ;{ }^{\mathrm{d}} \mathrm{P}=0.004$. EZH2, enhancer of zeste 2 polycomb repressive complex 2 subunit; EZH2-OE, EZH2-overexpressing.

A

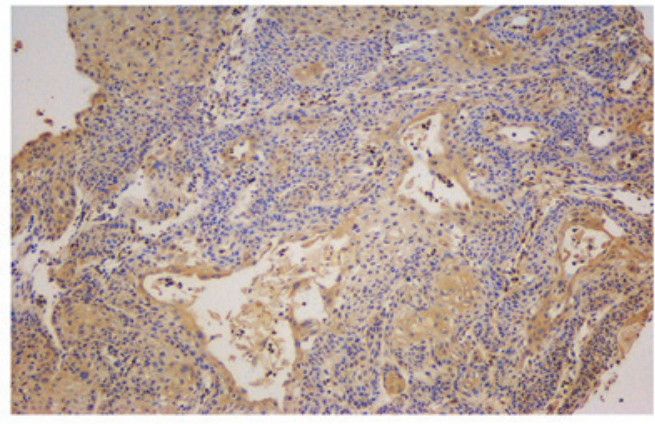

C

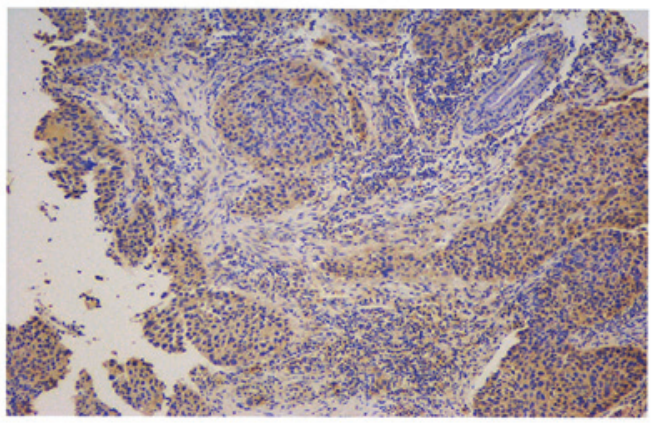

B

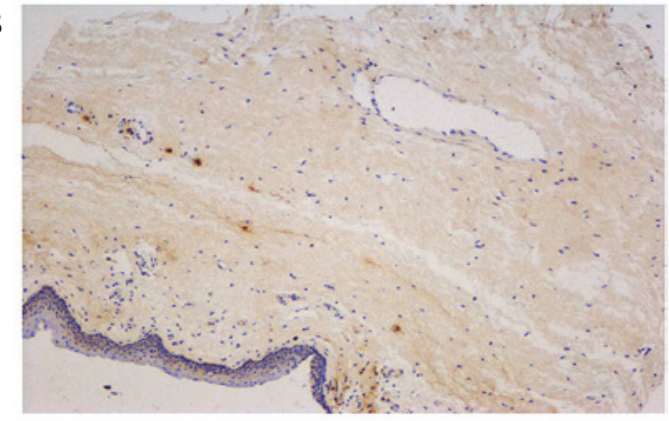

D

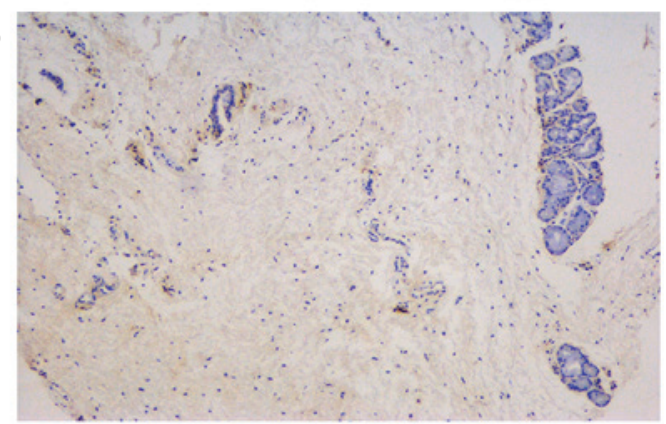

Figure 3. Expression of EZH2 in laryngeal squamous cell cancers. The tumor tissues and paracancerous tissues in the tissue microarray were stained using immunohistochemistry. (A and C) Representative and paracancerous tumor tissues, repectively; (B and D) Representative and paracancerous tumor tissues, repectively of another patient. The immunohistochemical staining was observed under a fluorescence microscope (magnification, x100). The level of EZH2 expression in the tumor tissues was significantly increased compared with the paracancerous tissues. EZH2, enhancer of zeste 2 polycomb repressive complex 2 subunit.

AMC-HN-8 cells, a proliferation assay was performed using a CCK-8. The results showed that the transfected cells exhibited an increased proliferative ability compared with the control group (Fig. 1D). In particular, the proliferative capacity of the transfected cells was significantly greater compared with the AMC-HN-8 cells between the fifth $(\mathrm{P}=0.012)$ to the seventh $(\mathrm{P}=0.004)$ of culture (Table II), suggesting that EZH2 overexpression enhanced the proliferative capacity of the AMC-HN-8 cells. To examine the effect of EZH2 overexpression on AMC-HN-8 cells, cell cycle and apoptosis analyses were conducted using flow cytometry. The cell cycle assay revealed that fewer EZH2-overexpressing cells had accumulated in the $\mathrm{G} 0-\mathrm{G} 1$ phase $(\mathrm{P}=0.001)$, but that more had accumulated in the synthesis phase $(\mathrm{P}=0.001)$ compared with the control cells (Fig. 4A-C). Subsequently, an apoptosis assay was performed by staining cells with anti-annexin-V and PI (Fig. 4D and E). The results showed that the average apoptosis rate of EZH2-overexpressing cells was $6.870 \pm 1.803 \%$, whereas the rate for the control group was $6.150 \pm 0.583 \%$ (Fig. 4F), and these rates were not significantly different $(\mathrm{P}=0.545)$.
EZH2 overexpression induces chemotherapy resistance. To evaluate the chemotherapy sensitivity of control and EZH2-overexpressing AMC-HN-8 cells, cisplatinum was applied to the cells and the rate of the tumor cell growth inhibition was determined. As shown in Fig. 5, the rate of growth inhibition of the two types of cells increased with the increasing dose of cisplatin. The rate of inhibition of the growth of the transfected tumor cells was significantly decreased compared with the control AMC-HN-8 cells, and the statistical analysis showed that the rates were significantly different in the $3 \mu \mathrm{g} / \mathrm{ml}(\mathrm{P}=0.027)$ and $6 \mu \mathrm{g} / \mathrm{ml}(\mathrm{P}=0.006)$ groups (Table III). Therefore, EZH2 overexpression increased the level of cisplatin resistance in AMC-HN-8 cells.

EZH2 overexpression promotes tumorigenesis in vivo. To investigate whether EZH2 overexpression affected tumorigenesis in vivo, NOD mice were used to perform a tumorigenesis assay. Fig. 6A shows an image of a representative mouse, 20 days subsequent to being injected with control and transfected AMC-HN-8 cells. Tumors were present in 

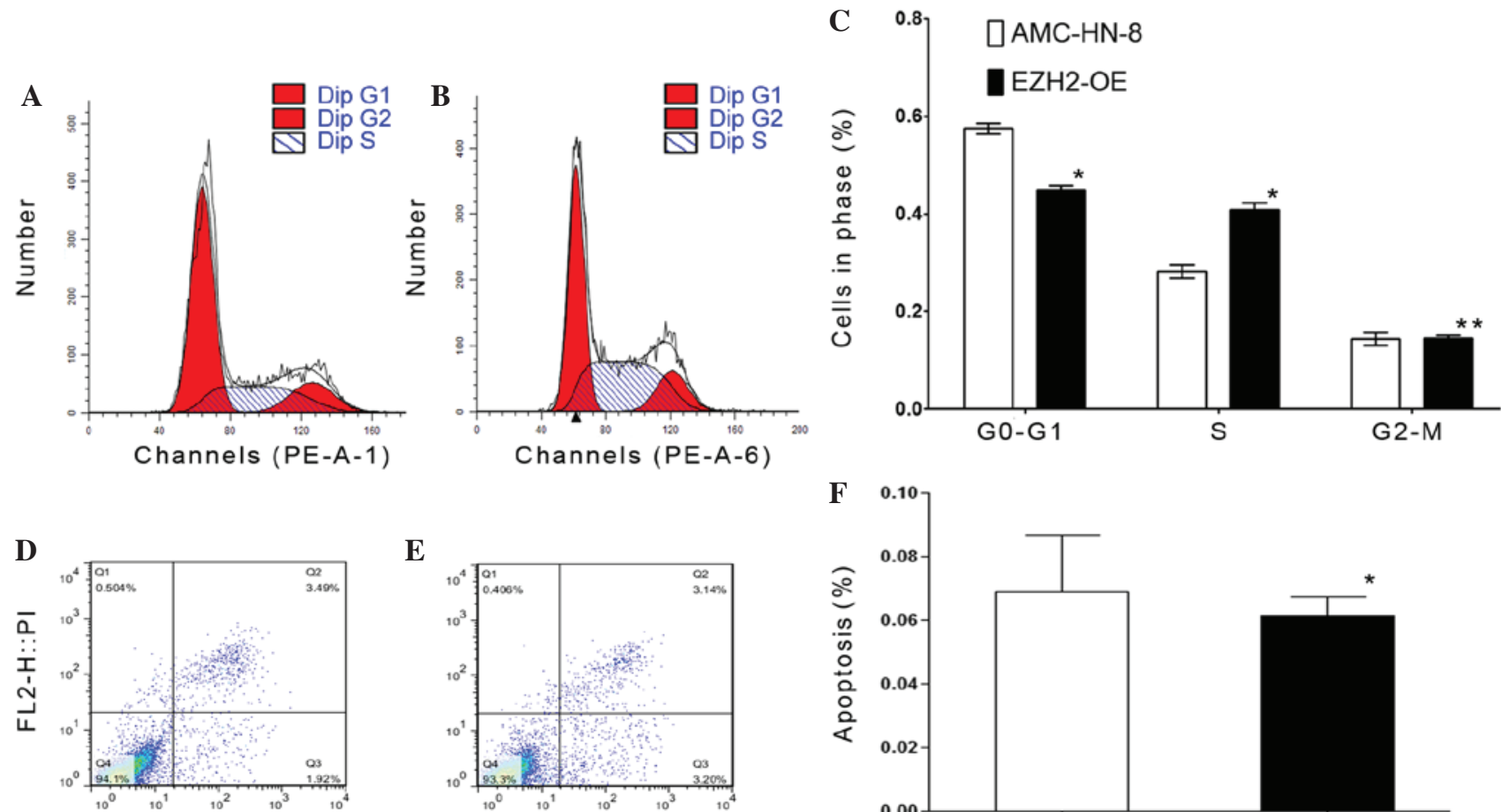

$\mathbf{E}$

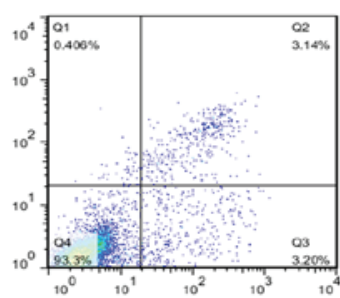

FL1-H::AnnexinV FITC

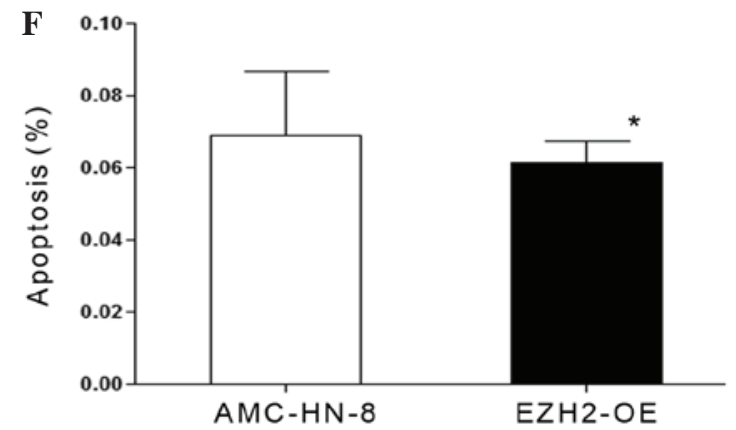

Figure 4. EZH2 overexpression induces AMC-HN-8 cells to pass the G0/G1 checkpoint, but has no effect on their apoptotic rate. Cell cycle analysis was performed using flow cytometry. (A) Representative percentages of AMC-HN-8 cells in the phases of the cell cycle with $57 \%$ of the cells in G0/G1 phase. (B) Representative percentages of EZH2-overexpressing cells in the phases of the cell cycle with $44 \%$ of the cells in the G0/G1 phase. (C) Comparison of the percentages of AMC-HN-8 cells and EZH2-overexpressing cells in each phase of the cell cycle. The apoptotic rate of these cells was determined using flow cytometry following staining with anti-annexin-V and PI. $\mathrm{P}<0.05,{ }^{* *} \mathrm{P}>0.05$. (D) Representative distribution of apoptotic and non-apoptotic AMC-HN-8 cells (6.88\% of these cells were apoptotic). (E) Representative distribution of apoptotic and non-apoptotic EZH2-overexpressing cells (6.15\% of these cells were apoptotic). (F) Comparison of the percentages of apoptotic AMC-HN-8 cells and EZH2-OE cells. The results were expressed as the mean values \pm standard deviation. ${ }^{*} \mathrm{P}<0.05$. EZH2, enhancer of zeste 2 polycomb repressive complex 2 subunit; EZH2-OE, EZH2-overexpressing; PI, propidium iodide; FITC, fluorescein isothiocyanate.

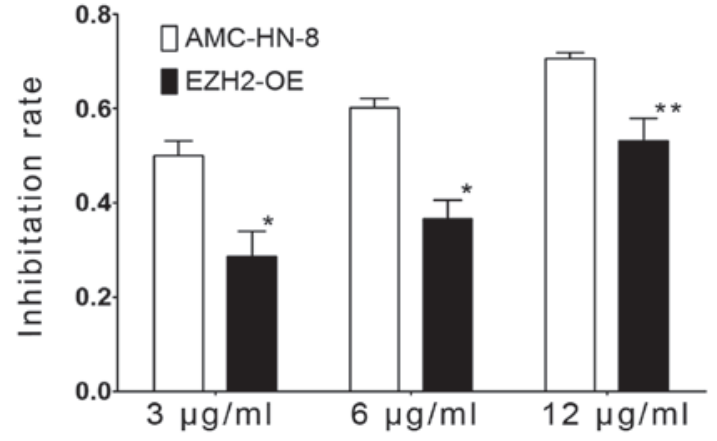

Figure 5. EZH2 overexpression increases the level of drug resistance of the AMC-HN-8 cells. AMC-HN-8 cells and EZH2-OE cells were treated with cisplatin at doses of 3,6 and $12 \mu \mathrm{g} / \mathrm{ml}$. After $24 \mathrm{~h}$, the number of viable cells was determined using the cell counting kit- 8 method, and the rate of growth inhibition was calculated. Results were expressed as the mean values \pm standard deviation. ${ }^{*} \mathrm{P}<0.05,{ }^{* *} \mathrm{P}>0.05$. EZH 2 , enhancer of zeste 2 polycomb repressive complex 2 subunit; EZH2-OE, EZH2-overexpressing.

each armpit of the mice. At 20 days post-injection, the mice were sacrificed, and the tumors were surgically removed. Fig. 6B shows images of the two types of tumors that grew in the mice. Fig. 6C shows that the tumors derived from the EZH2-overexpressing cells were significantly larger compared with those derived from the AMC-HN-8 cells. The mean tumor weight in the transfected group was greater compared with the AMC-HN-8 group, with values of
$0.2157 \pm 0.0256$ and $0.0780 \pm 0.0303 \mathrm{~g}$ for tumors derived from EZH2-overexpressing cells and the control AMC-HN-8 cells $(\mathrm{P}=0.001)$, respectively. Fig. 6D shows images of hematoxylin-eosin stained tumor sections. A professional pathologist determined that the histological characteristics of the sections were those of tumor tissues. In summary, EZH2 overexpression promoted the tumorigenesis of laryngeal squamous cell carcinoma cells in vivo.

\section{Discussion}

Patients with advanced stage laryngeal cancers demonstrate a low rate of successful treatment when treated using traditional therapies, including surgery, chemotherapy and radiotherapy (11). In order to optimize the effects of the traditional therapies, novel treatments, including gene-targeted therapies, are currently being explored. EZH2, a core catalytic subunit of PRC2, has been previously reported to mediate the proliferation and differentiation of hematopoietic (12), skeletal-muscle (13) and neural stem cells (14). In addition, $\mathrm{EZH} 2$ has been reported to be involved in sustaining the proliferative capacity and preventing the apoptosis of prostate cancer stem cell-like lines (6). EZH2 is highly expressed in numerous malignant tumors, including inflammatory breast cancer (15), lung cancer (16), renal cell carcinoma (17), cutaneous melanoma and prostate and endometrial cancer (18). $\mathrm{EZH} 2$ is also highly expressed in head and neck squamous 
Table III. Growth inhibition rates of AMC-HN-8 cells and EZH2-OE cells exposed to cisplatinum, a chemotherapeutic drug.

\begin{tabular}{lccc}
\hline & & Dose & \\
\cline { 2 - 4 } Type & $3 \mu \mathrm{g} / \mathrm{ml}^{\mathrm{a}}$ & $6 \mu \mathrm{g} / \mathrm{ml}^{\mathrm{b}}$ & $12 \mu \mathrm{g} / \mathrm{ml}^{\mathrm{c}}$ \\
\hline EZH2-OE & $0.4996 \pm 0.0540$ & $0.6022 \pm 0.0331$ & $0.7063 \pm 0.0201$ \\
AMC-HN-8 & $0.2856 \pm 0.0940$ & $0.3662 \pm 0.0680$ & $0.5305 \pm 0.0842$ \\
\hline
\end{tabular}

${ }^{\mathrm{a}} \mathrm{P}=0.027 ;{ }^{\mathrm{b}} \mathrm{P}=0.006 ;{ }^{\mathrm{C}} \mathrm{P}=0.055$. EZH2, enhancer of zeste 2 polycomb repressive complex 2 subunit; EZH2-OE, EZH2-overexpressing.

A

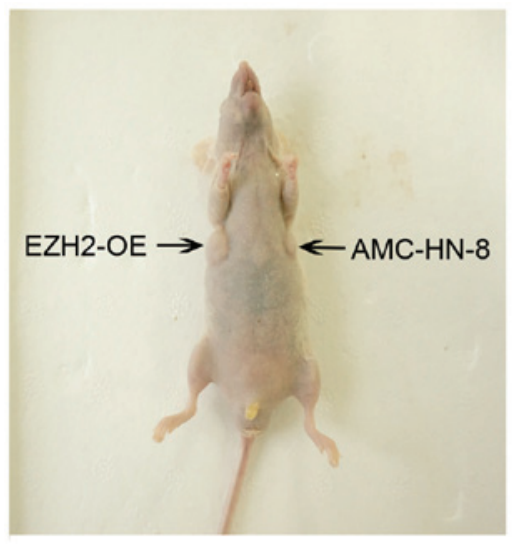

C

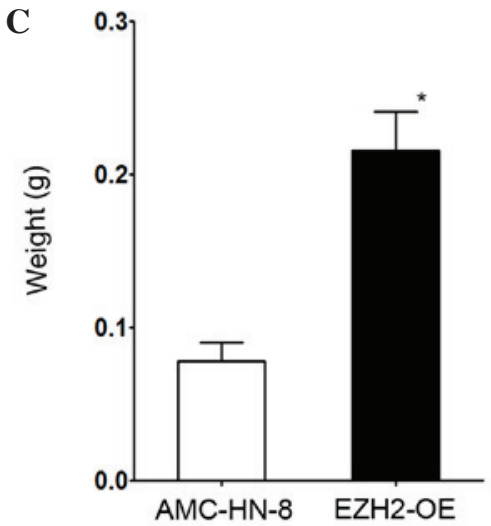

B

AMC-HN-8
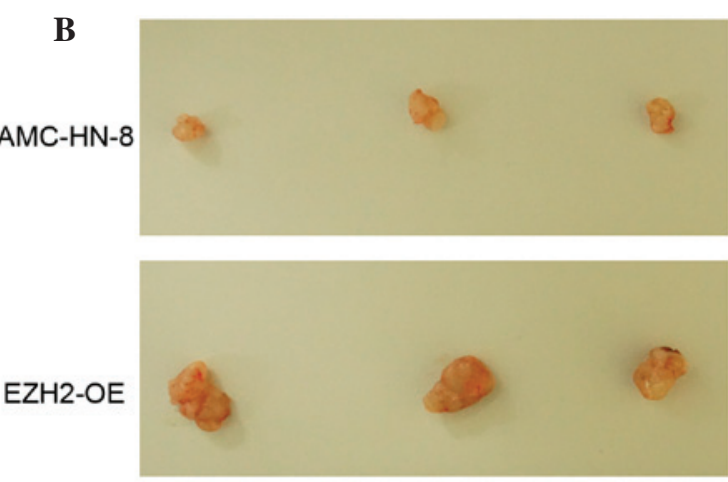

D
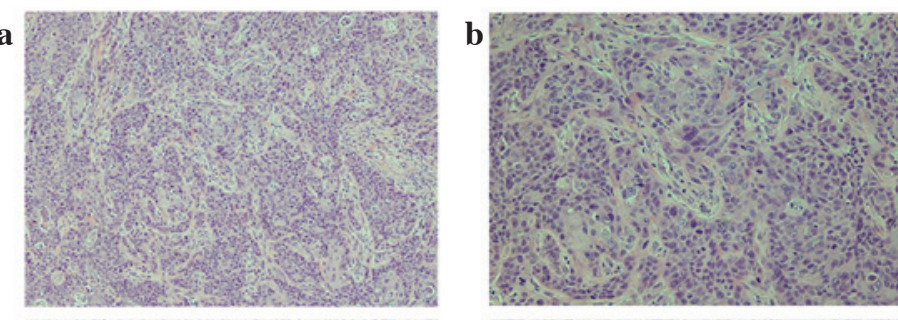

c
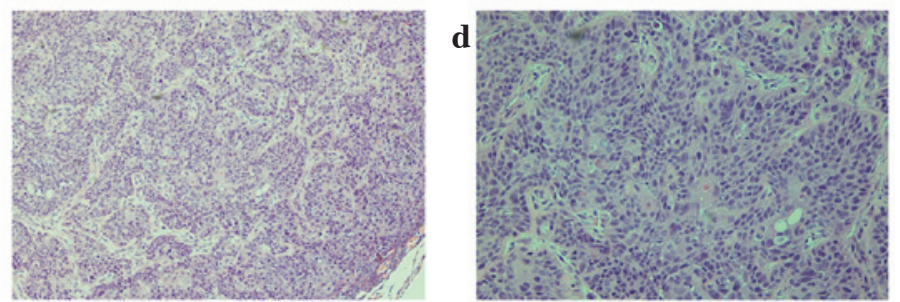

Figure 6. EZH2 overexpression promotes the in vivo tumorigenicity of AMC-HN-8 cells. (A) AMC-HN-8 cells and EZH2-OE cells were injected into NOD mice, and the resultant tumors were evaluated 20 days later. (B) Representative tumor masses that were removed from the sacrificed NOD mice. (C) Weights of the tumors derived from the AMC-HN-8 cells and EZH2-OE cells. " $\mathrm{P}<0.05$. (D) Tumor tissues were stained using hematoxylin-eosin and observed under a fluorescence microscope. The tumors exhibited the characteristics of squamous cell cancers. AMC-HN-8 group at (a) magnification, x100 and (b) magnification, x200. EZH2-OE group at (c) magnification, x100 and (d) magnification, x200. EZH2, enhancer of zeste 2 polycomb repressive complex 2 subunit; EZH2-OE, EZH2-overexpressing; NOD, non-obese diabetic.

cell cancers $(19,20)$. Kidani et al (19) reported that high-level EZH2 expression is associated with a poor prognosis of oral squamous cell cancers. Another study reported that EZH2 is highly expressed in certain nasopharyngeal carcinomas and is associated with a poor clinical outcome (20). These studies indicated that EZH2 may be a useful biomarker for making future clinical diagnoses and for predicting the clinical outcome of these diseases. The mechanisms of EZH2 have been examined in numerous studies, and to date, EZH2 has been reported to affect the proliferation, apoptosis, cell cycle and invasion of cancer cells (18-23). Therefore, EZH2 is categorized as an oncogene in certain types of tumors, which may influence pharmaceutical companies to develop a drug that specifically targets it. However, the expression level and function of EZH2 in laryngeal cancers is unknown. The present study revealed that the expression levels of EZH2 in primary laryngeal tumor tissues were increased compared with paracancerous epithelial tissues, and indicated that the upregulation of EZH2 expression promoted AMC-HN-8 cell proliferation in vitro by inducing the cells to pass the G0-G1 checkpoint. Notably, the present study demonstrated that $\mathrm{EZH} 2$ overexpression diminished sensitivity to cisplatin and facilitated the in vivo tumorigenicity of the cells.

In the present study, the expression of EZH2 was evaluated in specimens collected from patients that suffered from laryngeal cancers and had received surgery at the Eye, Ear, 
Nose and Throat Hospital of Fudan University. RT-qPCR and tissue microarray assays revealed that EZH2 was expressed at a significantly greater level in tumor tissues compared with the paracancerous epithelial tissues. However, unlike the findings regarding other polycomb-group proteins, such as BMI1 proto-oncogene, polycomb ring finger (BMI1) (24), the present study found that the increased expression level of EZH2 was associated with the location but not the stage of the tumors. In addition, glottic cancers had greater EZH2 expression levels compared with nonglottic cancers. However, the difference in the levels of EZH2 expression in tumors of various stages was not significant, the reasons for which are unknown and require further investigation. In the present study, clear differences were observed between the EZH2 expression levels of individual tumors in the same group, which indicated that the value of EZH2 as a clinical biomarker in laryngeal squamous cell cancers may be limited. Increased numbers of specimens must be examined in the future to expand the sample size and verify the results of the present study. The tissue microarrays may provide additional information, including potential correlations between the level of EZH2 expression and the 5-year survival rate, but this cannot be determined for a few years. In summary, the present study demonstrated that EZH2 was highly expressed in laryngeal cancers, which is consistent with previous findings regarding other types of cancer. However, the use of EZH2 expression as a diagnostic or prognostic factor requires additional assessment.

Several studies have reported that EZH2 is essential to the renewal and differentiation of numerous types of stem cells $(25,26)$. The enhanced expression of EZH2 allows these cells to more easily undergo malignant transformation. In contrast, the knockdown of EZH2 expression suppresses the proliferation of cancer cells in numerous malignancy models, including prostate cancer (6), breast cancer (27) and lymphoma (28). However, certain studies have reported that the knockdown of EZH2 and BMI1 expression does not prevent osteosarcoma cell proliferation (29). In the case of head and neck squamous cell cancers, certain studies have demonstrated that EZH2 affects cancer cell proliferation. For example, Zhao et al reported that the suppression of EZH2 expression reduces the proliferative ability of oral squamous cell carcinoma (10). In another study, Alajez et al (20) reported that the targeted depletion of EZH2 decreases the viability of nasopharyngeal carcinoma cells. These studies suggest that EZH2 may affect the stem cell-like properties of cancer cells. Therefore, the present tested whether this hypothesis was valid for laryngeal squamous cell carcinomas. EZH2 expression was upregulated through lentiviral transfection, and RT-qPCR and western blotting were used to verify the upregulation of EZH2. The findings demonstrated that the upregulation of EZH2 expression significantly enhanced the proliferation of AMC-HN-8 cells in vitro and in vivo. These results were consistent with those of previous reports regarding head and neck squamous cell cancers $(19,20)$.

Cell cycle progression and apoptosis affect the rate of cell proliferation. Previous studies have shown that EZH2 is important for the cell cycle and apoptosis by affecting other signaling pathways. Wu et al (30) reported that the depletion of EZH2 results in defective G1 and G2/M cell cycle checkpoints and that EZH2 depletion promotes apoptosis. Zhang et al (31) reported that the downregulation of EZH2 expression increases the rate of the docetaxel-induced apoptosis of prostate cancer cells. Thus, the present study used cell cycle and apoptosis assays to evaluate the effect of EZH2 overexpression. The results revealed that EZH2 overexpression shortened the G0-G1 phase, increased the percentage of cells entering $\mathrm{S}$ phase and induced more efficient cell proliferation. Consistent with the results of previous studies, the present results demonstrated that EZH2 affected the cell cycle. However, although EZH2 overexpression did not affect the rate of apoptosis, whether the suppression of EZH2 expression promotes apoptosis remains unknown.

Cancer stem cells are responsible for the chemotherapy resistance of tumors. EZH2 is a cancer stem cell-specific gene, so the effect of EZH2 on the chemotherapy resistance of tumors is worth studying (32). Hu et al (33) reported that EZH2 overexpression contributes to the acquired cisplatin resistance of ovarian cancer cells in vitro and vivo. Meng et al (34) reported that EZH2 overexpression is associated with a decreased 5-year survival rate of rectal cancer patients treated with neoadjuvant chemotherapy. Therefore, the cisplatin sensitivity of control and $\mathrm{EZH} 2$-overexpressing AMC-HN-8 cells was assessed in the present study. Consistent with the results of previous studies, the present findings showed that EZH2 had a similar effect on the drug resistance of laryngeal squamous cancer cells, indicating that $\mathrm{EZH} 2$ can enhance the drug resistance of cancer cells. This finding suggests that EZH2 may provide a novel approach for promoting the effect of chemotherapy on laryngeal cancer patients.

The results of the present study indicated that EZH2 may be an important target in laryngeal cancer stem cells. However, additional studies regarding the role of EZH2 in other properties of cancer stem cells are required. For example, studies have indicated that $\mathrm{EZH} 2$ promotes the invasion and angiogenesis of certain types of cancer cells $(21,35)$. In addition, in the preliminary experiments of the present study, the downregulation of EZH2 expression was observed to enhance the invasive ability of AMC-HN-8 cells. Thus, EZH2 could increase the invasive ability of laryngeal cancer cells, and high-level expression may result in a poor outcome for laryngeal cancer patients. The authors intend to investigate the association between EZH2 expression and the invasion of laryngeal cancer cells in the future. Since non-coding RNA has become a topic of intense research, various studies have focused on the association between EZH2 and non-coding RNA. Benetatos et al reported that non-coding RNAs affect the expression of protein-coding genes and establish feedback loops through interacting with EZH2 (36). Non-coding RNAs have also been indicated to participate in networks involving upstream and downstream factors that require EZH2 (36). Notably, a recent study indicated that the expression of several non-coding RNAs, including long non-coding RNAs CDKN2B antisense RNA 1, HOX transcript antisense RNA and metastasis associated lung adenocarcinoma transcript 1, was dramatically decreased with increasing concentrations of cisplatin and longer treatment durations in patients with laryngeal cancer, who received cisplatin therapy (37). Thus, EZH2 and several non-coding RNAs affect the level of chemotherapy resistance of tumors. Additional studies should therefore be performed 
in order to determine the involvement of these proteins and to investigate the relevance of the signaling pathways associated with this process.

In conclusion, the present study evaluated the association between EZH2 expression and laryngeal squamous cell cancers. EZH2 was demonstrated to be highly expressed in laryngeal squamous cell cancer tissues and to promote the proliferation of AMC-HN-8 cells in vitro and in vivo. Furthermore, the overexpression of $\mathrm{EZH} 2$ was found to accelerate the cell cycle of AMC-HN-8 cells and to enhance their resistance to cisplatin. Therefore, the present study demonstrated that $\mathrm{EZH} 2$ is a factor that regulates the proliferation of laryngeal squamous cancer cells and is a potential chemotherapeutic target for the treatment of such cancers.

\section{Acknowledgements}

The authors would like to thank Dr Zhang Duo for his assistance with the qPCR experiment. The present study was supported by the Shanghai Science and Technology Foundation, Shanghai, China (grant no., 08JC1404000).

\section{References}

1. Siegel R, Naishadham D and Jemal A: Cancer statistics, 2012. CA Cancer J Clin 62: 10-29, 2012.

2. Simon JA and Lange CA: Roles of the EZH2 histone methyltransferase in cancer epigenetics. Mutat Res 647: 21-29, 2008.

3. Vire E, Brenner C, Deplus R, Blanchon L, Fraga M, Didelot C, Morey L, Van Eynde A, Bernard D, Vanderwinden JM, et al: The Polycomb group protein EZH2 directly controls DNA methylation. Nature 439: 871-874, 2006.

4. Lee TI, Jenner RG, Boyer LA, Guenther MG, Levine SS, Kumar RM, et al: Control of developmental regulators by polycomb in human embryonic stem cells. Cell 125: 301-313, 2006.

5. Ezhkova E, Pasolli HA, Parker JS, Stokes N, Su IH, Hannon G, Tarakhovsky A and Fuchs E: Ezh2 orchestrates gene expression for the stepwise differentiation of tissue-specific stem cells Cell 136: 1122-1135, 2009.

6. Li KQ, Liu C, Zhou BF, Bi L, Huang H, Lin T and Xu K: Role of $\mathrm{EZH} 2$ in the growth of prostate cancer stem cells isolated from LNCaP cells. Int J Mol Sci 14: 11981-11993, 2013.

7. Rizzo S, Hersey JM, Mellor P, Dai W, Santos-Silva A, Liber D, Luk L, Titley I, Carden CP, Box G, Hudson DL, Kaye SB and Brown R: Ovarian cancer stem cell-like side populations are enriched following chemotherapy and overexpress EZH2. Mol Cancer Ther. 10: 325-335, 2011.

8. Sobin LH, Gospodarowicz MK and Wittekind C: UICC TNM classification of malignant tumours. 7th edition. Wiley-Blackwell; Oxford: pp. 63-72.2009

9. Shi Y, Gong HL, Zhou L, Tian J and Wang Y: CD24: A novel cancer biomarker in laryngeal squamous cell carcinoma. ORL J Otorhinolaryngol Relat Spec 74: 78-85, 2012.

10. Zhao LB, Yu Y, Wu J, Bai J, Zhao Y, Li C, Sun W and Wang X: Role of EZH2 in oral squamous cell carcinoma carcinogenesis. Gene 537: 197-202, 2014.

11. Blake JL, Runhua S, Vikas M, Glenn M, Federico A and Cherie-Ann ON: Improvements in survival and disparities for advanced-staged laryngeal cancer. JAMA Otolaryngol Head Neck Surg 141: 169-173, 2015.

12. Kamminga LM, Bystrykh LV, de Boer A, Houwer S, Douma J, Weersing E, Dontje B and de Haan G: The polycomb group gene Ezh2 prevents hematopoietic stem cell exhaustion. Blood 107: 2170-2179, 2006.

13. Juan AH, Derfoul A, Feng X, Ryall JG, Dell'Orso S, Pasut A, Zare H, Simone JM, Rudnicki MA and Sartorelli V: Polycomb EZH2 controls self-renewal and safeguards the transcriptional identity of skeletal muscle stem cells. Gene Dev 25: 789-794, 2011

14. Sher F, Rössler R, Brouwer N, Balasubramaniyan V, Boddeke E and Copray S: Differentiation of neural stem cells into oligodendrocytes: Involvement of the polycomb group protein Ezh2. Stem Cells 26: 2875-2883, 2008.
15. Gong Y, Huo L, Liu P, Sneige N, Sun X, Ueno NT, Lucci A, Buchholz TA, Valero V and Cristofanilli M: Polycomb group protein EZH2 is frequently expressed in inflammatory breast cancer and is predictive of worse clinical outcome. Cancer 117: 5476-5484, 2011.

16. Wan L, Li X, Shen H and Bai X: Quantitative analysis of EZH2 expression and its correlations with lung cancer patients' clinical pathological characteristics. Clin Transl Oncol 15: 132-138, 2013.

17. Wagener N, Macher-Goeppinger S, Pritsch M, Hüsing J, Hoppe-Seyler K, Schirmacher P, Pfitzenmaier J, Haferkamp A, Hoppe-Seyler F and Hohenfellner M: Enhancer of zeste homolog $2(\mathrm{EZH} 2)$ expression is an independent prognostic factor in renal cell carcinoma. Bmc Cancer 10: 524, 2010.

18. Bachmann IM, Halvorsen OJ, Collett K, Stefansson IM, Straume O, Haukaas SA, Salvesen HB, Otte AP, et al: EZH2 expression is associated with high proliferation rate and aggressive tumor subgroups in cutaneous melanoma and cancers of the endometrium, prostate and breast. J Clin Oncol 24: 268-273, 2006

19. Kidani K, Osaki M, Tamura T, Yamaga K, Shomori K, Ryoke K and Ito $\mathrm{H}$ : High expression of EZH2 is associated with tumor proliferation and prognosis in human oral squamous cell carcinomas. Oral Oncol 45: 39-46, 2009.

20. Alajez NM, Shi W, Hui AB, Bruce J, Lenarduzzi M, Ito E, Yue S, O'Sullivan B and Liu F: Enhancer of Zeste homolog 2 (EZH2) is overexpressed in recurrent nasopharyngeal carcinoma and is regulated by miR-26a, miR-101 and miR-98. Cell Death Dis 1: e85, 2010.

21. Rao ZY, Cai MY, Yang GF, He LR, Mai SJ, Hua WF, Liao YJ, Deng HX, Chen YC, Guan XY, et al: EZH2 supports ovarian carcinoma cell invasion and/or metastasis via regulation of TGF-betal and is a predictor of outcome in ovarian carcinoma patients. Carcinogenesis 31: 1576-1583, 2010.

22. Tong ZT, Cai MY, Wang XG, Kong LL, Mai SJ, Liu YH, Zhang HB, Liao YJ, Zheng F, Zhu W, et al: EZH2 supports nasopharyngeal carcinoma cell aggressiveness by forming a co-repressor complex with HDAC1/HDAC2 and Snail to inhibit E-cadherin. Oncogene 31: 583-594, 2012.

23. Crea F, Fornaro L, Bocci G, Sun L, Farrar WL, Falcone A and Danesi R: EZH2 inhibition: Targeting the crossroad of tumor invasion and angiogenesis. Cancer Metast Rev 31: 753-761, 2012.

24. Chen H, Zhou L, Wan G, Dou T and Tian J: BMI1 promotes the progression of laryngeal squamous cell carcinoma. Oral Oncol 47: 472-481, 2011.

25. Suvà ML, Riggi N, Janiszewska M, Radovanovic I, Provero $P$, Stehle JC, Baumer K, Le Bitoux MA, Marino D, Cironi L, et al: EZH2 Is Essential for glioblastoma cancer stem cell maintenance. Cancer Res 69: 9211-9218, 2009.

26. Majewski IJ, Blewitt ME, de Graaf CA, McManus EJ, Bahlo M, Hilton AA, Hyland CD, Smyth GK, Corbin JE, Metcalf D, et al: Polycomb repressive complex 2 (PRC2) restricts hematopoietic stem cell activity. PLoS Biol 6: e93, 2008.

27. van Vlerken LE, Kiefer CM, Morehouse C, Li Y, Groves C, Wilson SD, Yao Y, Hollingsworth RE and Hurt EM: EZH2 is required for breast and pancreatic cancer stem cell maintenance and can be used as a functional cancer stem cell reporter. Stem Cells Transl Med 2: 43-52, 2013.

28. McCabe MT, Ott HM, Ganji G, Korenchuk S, Thompson C, Van Aller GS, Liu Y, Graves AP, Della Pietra A III, Diaz E, et al: EZH2 inhibition as a therapeutic strategy for lymphoma with EZH2-activating mutations. Nature 492: 108-112, 2012.

29. Sasaki H, Setoguchi T, Matsunoshita Y, Gao H, Hirotsu M and Komiya S: The knock-down of overexpressed EZH2 and BMI-1 does not prevent osteosarcoma growth. Oncol Rep 23: 677-684, 2010.

30. Wu Z, Lee ST, Qiao Y, Li Z, Lee PL, Lee YJ, Jiang X, Tan J, Aau M, Lim CZ and Yu Q: Polycomb protein EZH2 regulates cancer cell fate decision in response to DNA damage. Cell Death Differ 18: 1771-1779, 2011.

31. Zhang Q, Padi SK, Tindall DJ and Guo B: Polycomb protein EZH2 suppresses apoptosis by silencing the proapoptotic miR-31. Cell Death Dis 5: e1486, 2014.

32. Serguei V and Xin W: Cancer stem cells and drug resistance: The potential of nanomedicine. Nanomedicine 7: 597-615, 2012.

33. Hu S, Yu L, Li Z, Shen Y, Wang J, Cai J, Xiao L and Wang Z: Overexpression of EZH2 contributes to acquired cisplatin resistance in ovarian cancer cells in vitro and in vivo. Cancer Biol Ther 10: 788-795, 2010. 
34. Meng X, Huang Z, Wang R, Jiao Y, Li H, Xu X, Feng R, Zhu K, Jiang S, Yan $\mathrm{H}$ and $\mathrm{Yu}$ J: The prognostic role of EZH2 expression in rectal cancer patients treated with neoadjuvant chemoradiotherapy. Radiat Oncol 9: 188, 2014.

35. Lu CH, Han HD, Mangala LS, Ali-Fehmi R, Newton CS, Ozbun L, Armaiz-Pena GN, Hu W, Stone RL, Munkarah A, et al: Regulation of tumor angiogenesis by EZH2. Cancer Cell 18: 185-197, 2010.
36. Benetatos L, Voulgaris E, Vartholomatos G and Hatzimichael E: Non-coding RNAs and EZH2 interactions in cancer: Long and short tales from the transcriptome. Int J Cancer 133: 267-274, 2013.

37. Chen H, Xin Y, Zhou L, Huang JM, Tao L, Cheng L and Tian J: Cisplatin and paclitaxel target significant long noncoding RNAs in laryngeal squamous cell carcinoma. Med Oncol 31: 246, 2014. 